Proceedings of the Edinburgh Mathematical Society (2005) 48, 479-484 (C)

DOI:10.1017/S001309150400032X Printed in the United Kingdom

\title{
INVARIANT SUBSPACES AND HANKEL-TYPE OPERATORS ON A BERGMAN SPACE
}

\author{
TAKAHIKO NAKAZI ${ }^{1}$ AND TOMOKO OSAWA ${ }^{2}$ \\ ${ }^{1}$ Department of Mathematics, Hokkaido University, Sapporo 060-0810, \\ Japan (nakazi@math.sci.hokudai.ac.jp) \\ ${ }^{2}$ Mathematical and Scientific Subjects, Asahikawa National College of Technology, \\ Asahikawa 071-8142, Japan (ohsawa@asahikawa-nct.ac.jp)
}

(Received 29 April 2004)

\begin{abstract}
Let $L^{2}=L^{2}(D, r d r d \theta / \pi)$ be the Lebesgue space on the open unit disc $D$ and let $L_{a}^{2}=L^{2} \cap \operatorname{Hol}(D)$ be a Bergman space on $D$. In this paper, we are interested in a closed subspace $\mathcal{M}$ of $L^{2}$ which is invariant under the multiplication by the coordinate function $z$, and a Hankel-type operator from $L_{a}^{2}$ to $\mathcal{M}^{\perp}$. In particular, we study an invariant subspace $\mathcal{M}$ such that there does not exist a finite-rank Hankel-type operator except a zero operator.
\end{abstract}

Keywords: Bergman space; invariant subspace; Hankel-type operator

2000 Mathematics subject classification: Primary 47B35; 47A15

\section{Introduction}

Let $D$ be the open unit disc in $\mathbb{C}$ and $\operatorname{Hol}(D)$ be the set of all holomorphic functions on $D$. Let $\mathrm{d} \mu=r d r d \theta / \pi$ and $L^{2}=L^{2}(D, \mathrm{~d} \mu)$ the Lebesgue space. The Bergman space $L_{a}^{2}$ on $D$ is defined by $L_{a}^{2}=L^{2} \cap \operatorname{Hol}(D)$. Then $L_{a}^{2}$ is the closed subspace of $L^{2}$. When $\mathcal{M}$ is a closed subspace of $L^{2}$ and $z \mathcal{M} \subseteq \mathcal{M}, \mathcal{M}$ is called an invariant subspace. For $\varphi$ in $L^{\infty}=L^{\infty}(D, \mathrm{~d} \mu)$, a Hankel-type operator is defined by

$$
H_{\varphi}^{\mathcal{M}} f=\left(I-P^{\mathcal{M}}\right)(\varphi f) \quad\left(f \in L_{a}^{2}\right),
$$

where $P^{\mathcal{M}}$ is the orthogonal projection from $L^{2}$ onto $\mathcal{M}$. When $\mathcal{M}=L_{a}^{2}, H_{\varphi}^{\mathcal{M}}$ is called a big Hankel operator and when $\mathcal{M}=\left(\bar{z} \overline{L_{a}^{2}}\right)^{\perp}, H_{\varphi}^{\mathcal{M}}$ is called a small Hankel operator. When $L_{a}^{2} \subseteq \mathcal{M} \subseteq\left(\bar{z} \overline{L_{a}^{2}}\right)^{\perp}, H_{\varphi}^{\mathcal{M}}$ is called an intermediate Hankel operator.

It is easy to see that there does not exist a finite-rank big Hankel operator except a zero one $($ see $[\mathbf{3}, \mathbf{6}])$. On the other hand, there exist a lot of finite-rank non-zero small Hankel operators (see [6]). In fact, it is easy to see the results. Strouse [7] described completely all finite-rank intermediate Hankel operators for some invariant subspace. In the previous paper [6], we began to study finite-rank intermediate Hankel operators for arbitrary invariant subspace. In [6, Theorem 3.2], we gave three necessary and sufficient 
conditions for $\mathcal{M}$ such that there does not exist a finite-rank intermediate Hankel operator except a zero one. In this paper, without the hypothesis on an invariant subspace $\mathcal{M}$, we give a new necessary and sufficient condition for $\mathcal{M}$ which have a finite-rank Hankel-type operator except a zero one.

For an invariant subspace $\mathcal{M}$ in $L^{2}, \operatorname{ker} H_{\varphi}^{\mathcal{M}}$ denotes the kernel of $H_{\varphi}^{\mathcal{M}}$ and then $\operatorname{ker} H_{\varphi}^{\mathcal{M}}=\left\{f \in L_{a}^{2} ; \varphi f \in \mathcal{M}\right\}$. Hence $\operatorname{ker} H_{\varphi}^{\mathcal{M}}$ is also an invariant subspace in $L_{a}^{2}$. Thus each invariant subspace $\mathcal{M}$ in $L^{2}$ is related to an invariant subspace in $L_{a}^{2}$ by a Hankeltype operator. In this paper, the following property of invariant subspaces in $L^{2}$ is important.

Definition 1.1. Let $\mathcal{M}$ be an invariant subspace of $L^{2} . \mathcal{M}$ is called weakly divisible if whenever $f \in \mathcal{M}$ and $|f(z)| \leqslant \gamma|z-a|$ for some $a \in D$ and some $\gamma \geqslant 0$ then $f(z)=(z-a) g(z)$ and $g$ is a function in $\mathcal{M}$.

In $\S 2$, we generalize a theorem of Axler and Bourdon [1] , which will be used later on. In $\S 3$, we show that there does not exist a finite-rank Hankel-type operator $H_{\varphi}^{\mathcal{M}}$ except a zero one if and only if $\mathcal{M}$ is weakly divisible. In $\S 4$, we give several examples of weakly divisible invariant subspaces.

In this paper $[S]_{*}$ denotes the weak* closed linear span of a subset $S$ in $L^{\infty}$ and $[S]_{2}$ denotes the closed linear span of a subset $S$ in $L^{2}$.

\section{An invariant subspace and the index}

In this section, for a given invariant subspace $\mathcal{M}$ we are interested in two invariant subspaces $\mathcal{M}^{\prime}$ and $\mathcal{M}^{\prime \prime}$ such that $\mathcal{M}^{\prime} \subseteq \mathcal{M} \subseteq \mathcal{M}^{\prime \prime}$, $\operatorname{dim} \mathcal{M} \ominus \mathcal{M}^{\prime}<\infty$ and $\operatorname{dim} \mathcal{M}^{\prime \prime} \ominus \mathcal{M}<$ $\infty$. Under some conditions on $\mathcal{M}, \mathcal{M}^{\prime}$ and $\mathcal{M}^{\prime \prime}$, we describe $\mathcal{M}^{\prime}$ and $\mathcal{M}^{\prime \prime}$ using $\mathcal{M}$. Corollary 2.4 will be used in $\S \S 3$ and 4 . Corollary 2.4 (i) is known from [1] .

When $\mathcal{M}$ is an invariant subspace of $L^{2}$, for $a \in \mathbb{C}$ put $\operatorname{ind}_{a} \mathcal{M}=\operatorname{dim}\{\mathcal{M} \ominus(z-a) \mathcal{M}\}$. $\operatorname{ind}_{a} \mathcal{M}$ is called the index of $\mathcal{M}$ at $a$. It is known (cf. [1]) that for each $n(0 \leqslant n \leqslant \infty)$ and for any $a(\in D)$ there exists an invariant subspace $\mathcal{M}$ with $\operatorname{ind}_{a} \mathcal{M}=n$.

Theorem 2.1. Let $\mathcal{M}, \mathcal{M}_{1}$ and $\mathcal{M}_{2}$ be invariant subspaces of $L^{2}$ and $\mathcal{M}_{1} \subseteq \mathcal{M}_{2}$.

(i) $\operatorname{ind}_{a} \mathcal{M}=0$ for any $a \notin D$.

(ii) If $\operatorname{dim} \mathcal{M}_{2} \ominus \mathcal{M}_{1}<\infty$, then there exists a polynomial $b$ such that $b \mathcal{M}_{2} \subseteq \mathcal{M}_{1}$, $Z(b) \subset D$ and the degree of $b \leqslant \operatorname{dim} \mathcal{M}_{2} \ominus \mathcal{M}_{1}$ and

$$
\sum\left(\operatorname{ind}_{a} \mathcal{M}_{2} ; a \in Z(b)\right) \geqslant \operatorname{dim} \mathcal{M}_{2} \ominus \mathcal{M}_{1} .
$$

Proof. (i) If $|a|>1$, then $(z-a)^{-1} \in H^{\infty}$ and $\mathcal{M}=(z-a) \mathcal{M}$. Hence $\operatorname{ind}_{a} \mathcal{M}=0$. If $|a|=1$, then $(z-a) \mathcal{M}=(z-a)\{z-a(1+\varepsilon)\}^{-1} \mathcal{M}$. For any $f \in \mathcal{M}$, it is easy to see that

$$
\int_{D}\left|\frac{z-a}{z-a(1+\varepsilon)} f-f\right|^{2} \mathrm{~d} \mu \rightarrow 0 \quad(\varepsilon \rightarrow 0)
$$

by Lebesgue's convergence theorem. This implies that $(z-a) \mathcal{M}$ is dense in $\mathcal{M}$ and so $\operatorname{ind}_{a} \mathcal{M}=0$ for $|a|=1$. 
(ii) Put $\mathcal{N}=\mathcal{M}_{2} \ominus \mathcal{M}_{1}$ and $\mathcal{S}_{z}=P M_{z} \mid \mathcal{N}$, where $M_{z}$ is a multiplication operator on $L^{2}$ by the coordinate function $z$ and $P$ is the orthogonal projection from $L^{2}$ to $\mathcal{N}$. If $n=\operatorname{dim} \mathcal{N}<\infty$, then there exists a polynomial $b$ of degree $n$ such that $\mathcal{S}_{b}=$ $b\left(\mathcal{S}_{z}\right)=0$ and so $b \mathcal{M}_{2} \subseteq \mathcal{M}_{1}$. By (i), we may assume that $Z(b) \subset D$. We will prove that $\sum\left(\operatorname{ind}_{a} \mathcal{M}_{2} ; a \in Z(b)\right) \geqslant n$. We can write that $b=a_{0} \prod_{j=1}^{n}\left(z-a_{j}\right)$ and so $Z(b)=$ $\left\{a_{1}, a_{2}, \ldots, a_{n}\right\}$, where $a_{0} \in \mathbb{C}$. If $\sum\left(\operatorname{ind}_{a} \mathcal{M}_{2} ; a \in Z(b)\right) \leqslant n-1$, then we may assume $\operatorname{ind}_{a_{1}} \mathcal{M}_{2}=0$. Since $\left[\left(z-a_{1}\right) \mathcal{M}_{2}\right]_{2}=\mathcal{M}_{2}$,

$$
\prod_{j=2}^{n}\left(z-a_{j}\right) \mathcal{M}_{2} \subseteq \mathcal{M}_{1} \subset \mathcal{M}_{2} .
$$

Then it is easy to see that $\operatorname{dim} \mathcal{M}_{2} \ominus\left[\prod_{j=2}^{n}\left(z-a_{j}\right) \mathcal{M}_{2}\right]_{2} \leqslant n-1$ because $\operatorname{ind}_{a_{j}} \mathcal{M}_{2} \leqslant 1$ for $2 \leqslant j \leqslant n$. This contradicts that $\operatorname{dim} \mathcal{M}_{2} \ominus \mathcal{M}_{1}=n$.

Corollary 2.2. Let $\mathcal{M}_{1}$ and $\mathcal{M}_{2}$ be invariant subspaces of $L^{2}$ and $\mathcal{M}_{1} \subseteq \mathcal{M}_{2}$. If $\operatorname{dim} \mathcal{M}_{2} \ominus \mathcal{M}_{1}=1$, then $(z-a) \mathcal{M}_{2} \subseteq \mathcal{M}_{1} \subsetneq \mathcal{M}_{2}$ for some $a \in D$ and $\operatorname{ind}_{a} \mathcal{M}_{2} \geqslant 1$. If $\operatorname{ind}_{a} \mathcal{M}_{1}=1$ or $\operatorname{ind}_{a} \mathcal{M}_{2}=1$, then $\mathcal{M}_{1}=\left[(z-a) \mathcal{M}_{2}\right]_{2}$.

Proof. By Theorem 2.1, $(z-a) \mathcal{M}_{2} \subseteq \mathcal{M}_{1}$ for some $a \in D$ and so ind $\mathcal{M}_{2} \geqslant 1$. Since $(z-a) \mathcal{M}_{1} \subseteq(z-a) \mathcal{M}_{2} \subseteq \mathcal{M}_{1} \subsetneq \mathcal{M}_{2}, \mathcal{M}_{1}=\left[(z-a) \mathcal{M}_{2}\right]_{2}$ if $\operatorname{ind}_{a} \mathcal{M}_{1}=1$ or $\operatorname{ind}_{a} \mathcal{M}_{2}=1$.

Corollary 2.3. Let $\mathcal{M}_{1}$ and $\mathcal{M}_{2}$ be invariant subspaces such that $\mathcal{M}_{1} \subsetneq \mathcal{M}_{2}$ and $\operatorname{dim} \mathcal{M}_{2} \ominus \mathcal{M}_{1}=n<\infty$. Suppose that $(z-a) \mathcal{M}_{j}$ is closed for any $a$ in $D$ when $j=1,2$. If $\operatorname{ind}_{a} \mathcal{M}_{1}=1$ for any $a$ in $D$ or $\operatorname{ind}_{a} \mathcal{M}_{2}=1$ for any $a$ in $D$, then $\mathcal{M}_{1}=b \mathcal{M}_{2}$ and $\mathcal{M}_{2}=\left\langle f_{1} / b, \ldots, f_{n} / b\right\rangle \oplus \mathcal{M}_{1}$, where $b=\prod_{j=1}^{n}\left(z-a_{j}\right),\left\{a_{j}\right\} \subset D$ and $\left\{f_{j}\right\} \subset \mathcal{M}_{1}$.

Proof. By Theorem 2.1 there exists a polynomial $b$ such that $b \mathcal{M}_{2} \subseteq \mathcal{M}_{1}$ and $Z(b) \subset$ $D$ and the degree of $b \leqslant n$. Hence $b=\prod_{j=1}^{\ell}\left(z-a_{j}\right)$ and $\left\{a_{j}\right\} \subset D$ and $\ell \leqslant n$. When $\operatorname{ind}_{a} \mathcal{M}_{2}=1$ for any $a$ in $D, \operatorname{dim} \mathcal{M}_{2} \ominus b \mathcal{M}_{2}=\ell$ because $\left(z-a_{j}\right) \mathcal{M}_{2}$ is closed for $1 \leqslant j \leqslant \ell$ and so $\ell=n$. Hence $\mathcal{M}_{1}=b \mathcal{M}_{2}$. When $\operatorname{ind}_{a} \mathcal{M}_{1}=1$ for any $a$ in $D, \operatorname{dim} \mathcal{M}_{1} \ominus b \mathcal{M}_{1}=\ell$ by the same reason. Since $b \mathcal{M}_{1} \subseteq b \mathcal{M}_{2} \subseteq \mathcal{M}_{1}$ and $\operatorname{dim} b \mathcal{M}_{2} \ominus b \mathcal{M}_{1}=n, \ell=n$ and so $\mathcal{M}_{1}=b \mathcal{M}_{2}$. Put $\mathcal{M}_{2}=\left\langle\varphi_{1}, \ldots, \varphi_{n}\right\rangle \oplus \mathcal{M}_{1}$, where $\left\{\varphi_{j}\right\}$ are orthogonal to $\mathcal{M}_{1}$. What was just proved above, $b \mathcal{M}_{2}=\mathcal{M}_{1}$ and so $b \mathcal{M}_{2}=\left\langle b \varphi_{1}, \ldots, b \varphi_{n}\right\rangle \oplus b \mathcal{M}_{1}=\mathcal{M}_{1}$. Put $f_{j}=b \varphi_{j}$ for $j=1, \ldots, n$, then $\left\{f_{j}\right\}$ are in $\mathcal{M}_{1}$ and $\mathcal{M}_{2}=\left\langle f_{1} / b, \ldots, f_{n} / b\right\rangle \oplus \mathcal{M}_{1}$.

Corollary 2.4. Let $\mathcal{M}$ be an invariant subspace of $L^{2}$.

(i) If $\operatorname{dim} L_{a}^{2} \ominus \mathcal{M}=n<\infty$ and $n \neq 0$, then $\mathcal{M}=b L_{a}^{2}$, where $b=\prod_{j=1}^{n}\left(z-a_{j}\right)$ and $\left\{a_{j}\right\} \subset D$.

(ii) If $\operatorname{dim} \mathcal{M} \ominus L_{a}^{2}=n<\infty$, then $\mathcal{M}=L_{a}^{2}$.

Proof. It is known that $\operatorname{ind}_{a} L_{a}^{2}=1$ and $(z-a) L_{a}^{2}$ is closed for each $a \in D$. Hence we can apply Corollary 2.3 to $\mathcal{M}_{1}=L_{a}^{2}$ or $\mathcal{M}_{2}=L_{a}^{2}$. If $\mathcal{M}_{1}=\mathcal{M}$ and $\mathcal{M}_{2}=L_{a}^{2}$, then (i) follows. If $\mathcal{M}_{1}=L_{a}^{2}$ and $\mathcal{M}_{2}=\mathcal{M}$, then $\mathcal{M}=\left\langle f_{1} / b, \ldots, f_{n} / b\right\rangle \oplus L_{a}^{2}$, where $b=\prod_{j=1}^{n}\left(z-a_{j}\right),\left\{a_{j}\right\} \subset D$ and $\left\{f_{j}\right\} \subset L_{a}^{2}$. For each $1 \leqslant \ell \leqslant n, f_{\ell} / b \in L^{2}$ and so 
$f_{\ell}\left(a_{j}\right)=0$ for $1 \leqslant j \leqslant n$. Then $f_{\ell} / b$ belongs to $L_{a}^{2}$ and so $f_{\ell} / b=0$ for each $\ell$. Thus $\mathcal{M}=L_{a}^{2}$ and so (ii) follows.

\section{Finite-rank Hankel-type operators}

In this section, we study the relation between finite-rank Hankel-type operators and invariant subspaces.

Theorem 3.1. Let $\mathcal{M}$ be an invariant subspace of $L^{2}$. Then there does not exist a finite-rank Hankel-type operator $H_{\varphi}^{\mathcal{M}}$ except a zero one if and only if $\mathcal{M}$ is weakly divisible.

Proof. Suppose $\mathcal{M}$ is weakly divisible. If $H_{\varphi}^{\mathcal{M}}$ is of finite rank, then $\operatorname{ker} H_{\varphi}^{\mathcal{M}}$ is an invariant subspace in $L_{a}^{2}$ and $\operatorname{dim} L_{a}^{2} / \operatorname{ker} H_{\varphi}^{\mathcal{M}}<\infty$. By (i) of Corollary 2.4, $\operatorname{ker} H_{\varphi}^{\mathcal{M}}=b L_{a}^{2}$ for some polynomial $b$ with $Z(b) \subset D$ and so $b \varphi$ belongs to $\mathcal{M}$. Put $f=b \varphi$, then $|f(z)| \leqslant \gamma|b(z)|(z \in D)$, where $\gamma=\|\varphi\|_{\infty}$. Suppose $b(z)=a_{0} \prod_{j=1}^{n}\left(z-a_{j}\right)$, where $\left\{a_{j}\right\} \subset D$. For any $\ell$ with $1 \leqslant \ell \leqslant n$,

$$
\left|\frac{f(z)}{z-a_{\ell}}\right| \leqslant \gamma\left|a_{0}\right| \prod_{j \neq \ell}\left|z-a_{j}\right| \quad(z \in D)
$$

and $f(z) /\left(z-a_{\ell}\right)$ belongs to $\mathcal{M}$ because $a_{\ell} \in D$ and $\mathcal{M}$ is weakly divisible. Thus $\varphi(z)=f(z) / b(z)$ belongs to $\mathcal{M}$. Hence $H_{\varphi}^{\mathcal{M}}=0$.

Conversely, if $\mathcal{M}$ is not weakly divisible, then there exists a function $f$ in $\mathcal{M}$ and a point $a$ in $D$ such that $|f(z)| \leqslant \gamma|z-a|(z \in D)$ and $f(z) /(z-a)$ does not belong to $\mathcal{M}$. Put $\varphi=f(z) /(z-a)$, then $\varphi \in L^{\infty}$ and $H_{\varphi}^{\mathcal{M}}$ is not zero because $\varphi \notin \mathcal{M}$. On the other hand, $(z-a) \varphi \in \mathcal{M}$ and so the kernel of $H_{\varphi}^{\mathcal{M}}$ contains $(z-a) L_{a}^{2}$. This implies that $H_{\varphi}^{\mathcal{M}}$ is of rank one because $L_{a}^{2} /(z-a) L_{a}^{2}=\mathbb{C}$.

Proposition 3.2. If there exists a symbol $\varphi$ such that $r\left(H_{\varphi}^{\mathcal{M}}\right)=n \geqslant 1$, then there exists a symbol $\varphi_{j}$ such that $r\left(H_{\varphi_{j}}^{\mathcal{M}}\right)=j$ for any $j$ with $0 \leqslant j \leqslant n-1$.

Proof. Suppose $1 \leqslant n=r\left(H_{\varphi}^{\mathcal{M}}\right)<\infty$. Then $\operatorname{ker} H_{\varphi}^{\mathcal{M}}=$ the kernel of $H_{\varphi}^{\mathcal{M}}$ is an invariant subspace of $L_{a}^{2}$ and $L_{a}^{2} / \operatorname{ker} H_{\varphi}^{\mathcal{M}}$ is of finite dimension $n$. By Corollary 2.4, $\operatorname{ker} H_{\varphi}^{\mathcal{M}}=b L_{a}^{2}$, where $b=\prod_{\ell=1}^{n}\left(z-a_{\ell}\right)$ and $\left(a_{\ell}\right) \subset D$. Hence $b \varphi$ belongs to $\mathcal{M}$. Put

$$
\varphi_{j}=\varphi \prod_{\ell=j+1}^{n}\left(z-a_{\ell}\right) \quad \text { for } 1 \leqslant j \leqslant n-1,
$$

then $\varphi_{j} \notin \mathcal{M}$ for $1 \leqslant j \leqslant n-1$ and $\varphi_{0}=b \varphi$. Since ker $H_{\varphi_{j}}^{\mathcal{M}}=b_{j} L_{a}^{2}$ for $1 \leqslant j \leqslant n-1$, where $b_{j}=\prod_{\ell=1}^{j}\left(z-a_{\ell}\right), H_{\varphi_{j}}^{\mathcal{M}}$ is of finite rank $j$ for $0 \leqslant j \leqslant n-1$.

Corollary 3.3. The following two expressions are equivalent for an invariant subspace $\mathcal{M}$.

(i) If $r\left(H_{\varphi}^{\mathcal{M}}\right)<\infty$, then $r\left(H_{\varphi}^{\mathcal{M}}\right)=0$. 
(ii) If $r\left(H_{\varphi}^{\mathcal{M}}\right) \leqslant 1$, then $r\left(H_{\varphi}^{\mathcal{M}}\right)=0$.

Proof. (i) $\Rightarrow$ (ii). This is clear.

(ii) $\Rightarrow$ (i). If (i) is not true, then there exists a symbol $\varphi$ with $r\left(H_{\varphi}^{\mathcal{M}}\right)=n \geqslant 2$. By Proposition 3.2 there exists a symbol $\varphi_{1}$ such that $r\left(H_{\varphi_{1}}^{\mathcal{M}}\right)=1$. This contradicts (ii).

\section{Weakly divisible invariant subspaces}

For a function $f$ in $L_{a}^{2}$, put $Z(f)=\{a \in D ; f(a)=0\}$ and $Z(G)=\cap\{Z(f) ; f \in G\}$ for a subset $G$ in $L_{a}^{2}$. For $1 \leqslant p \leqslant \infty$, if $E$ is an open set in $D, H_{E}^{p}$ denotes the set of all functions in $L^{p}$ that are analytic on $E$. In Corollary 4.2, a weakly divisible invariant subspace $\mathcal{M}$ is described completely when $\mathcal{M}$ is in $L_{a}^{2}$. There exists a non-zero invariant subspace $\mathcal{M}$ in $L_{a}^{2}$ such that $\mathcal{M} \cap L^{\infty}=\langle 0\rangle$. For it is known (see [5]) that there exists a non-zero function $f$ in $L_{a}^{2}$ such that $Z(f)$ does not satisfy the Blaschke condition.

Theorem 4.1. Let $\mathcal{M}$ be an invariant subspace of $L^{2}$.

(i) If $\mathcal{M} \cap L^{\infty} \subseteq H^{\infty}$ and $Z\left(\mathcal{M} \cap L^{\infty}\right)=\emptyset$, then $\mathcal{M}$ is weakly divisible.

(ii) If $\mathcal{M} \cap L^{\infty}=H_{E}^{\infty}$ for some open set $E$, then $\mathcal{M}$ is weakly divisible.

(iii) If $\mathcal{M} \cap L^{\infty}=\langle 0\rangle$, then $\mathcal{M}$ is weakly divisible.

Proof. (i) If $\left\{f_{n}\right\}$ is a sequence in $\mathcal{M} \cap L^{\infty}$ which converges pointwise boundedly to $f$, then $f \in \mathcal{M}$. By the Krein-Schmulian criterion (see [4, IV 2.1]), $\mathcal{M} \cap L^{\infty}$ is weak* closed. Hence, by a well-known theorem of Beurling [2] $\mathcal{M} \cap L^{\infty}=q H^{\infty}$ for some inner function $q$. Hence if $f \in \mathcal{M}$ and $|f(z)| \leqslant \gamma|z-a|(z \in D)$ for some $a \in D$, then $f=q h$ for some $h \in H^{\infty}$. Since $Z\left(\mathcal{M} \cap L^{\infty}\right)=\emptyset,|q(z)|>0(z \in D)$ and so $h(a)=0$. Hence $f(z) /(z-a)=q(z) \times(h(z) /(z-a)) \in q H^{\infty}$. Thus $f(z) /(z-a)$ belongs to $\mathcal{M}$.

(ii) If $f \in H_{E}^{\infty}$ and $|f(z)| \leqslant \gamma|z-a|(z \in D)$ for some $a \in D$, then $f(z) /(z-a) \in L^{\infty}$ and $f(z) /(z-a)$ is analytic on $E$. Hence $f(z) /(z-a)$ belongs to $H_{E}^{\infty}$ and so $\mathcal{M}$ is weakly divisible.

(iii) This is clear.

Corollary 4.2. Let $\mathcal{M}$ be an invariant subspace of $L_{a}^{2}$. Then $\mathcal{M}$ is weakly divisible if and only if $\mathcal{M} \cap L^{\infty}=\langle 0\rangle$ or $Z\left(\mathcal{M} \cap L^{\infty}\right)=\emptyset$.

Proof. The part of 'if' is a result of (i) and (iii) of Theorem 4.1. Conversely, suppose that $\mathcal{M}$ is weakly divisible. If $\mathcal{M} \cap L^{\infty} \neq\langle 0\rangle$, then by a theorem of Beurling there exists an inner function $q$ with $\mathcal{M} \cap L^{\infty}=q H^{\infty}$. If $q(a)=0$ for some $a \in D$, then there exists a finite positive constant $\gamma$ such that $|q(z)| \leqslant \gamma|z-a|(z \in D)$ and $q /(z-a) \notin \mathcal{M}$. This contradicts the weak divisibility of $\mathcal{M}$ and so $Z(q)=Z\left(\mathcal{M} \cap L^{\infty}\right)=\emptyset$.

Corollary 4.3. Let $\mathcal{M}$ be an invariant subspace of $L^{2}$.

(i) If $\mathcal{M} \subsetneq L_{a}^{2}$ and $\operatorname{dim} L_{a}^{2} / \mathcal{M}<\infty$, then $\mathcal{M}$ is not weakly divisible. 
(ii) If $\mathcal{M} \supseteq L_{a}^{2}$ and $\operatorname{dim} \mathcal{M} / L_{a}^{2}<\infty$, then $\mathcal{M}$ is weakly divisible.

Proof. (i) If $\mathcal{M} \subsetneq L_{a}^{2}$ and $\operatorname{dim} L_{a}^{2} / \mathcal{M}=\ell<\infty$, then by (i) of Corollary $2.4 \mathcal{M}=b L_{a}^{2}$, where $b=\prod_{j=1}^{\ell}\left(z-a_{j}\right)$ and $a_{j} \in D(1 \leqslant j \leqslant \ell)$. Hence $Z\left(\mathcal{M} \cap L^{\infty}\right)=Z(b) \neq \emptyset$ and so by Corollary $4.2 \mathcal{M}$ is not weakly divisible.

(ii) By (2) of Corollary 2.4 $\mathcal{M}=L_{a}^{2}$ and so $\mathcal{M} \cap L^{\infty}=H^{\infty}$. Hence (i) of Theorem 4.1 implies that $\mathcal{M}$ is weakly divisible.

Corollary 4.4. If $\mathcal{M}=H_{E}^{2}$ for some open set $E$ in $D$, then $\mathcal{M}$ is weakly divisible.

Proof. It is a result of (ii) of Theorem 4.1.

Proposition 4.5. Suppose that $\mathcal{M}_{j}$ is a weakly divisible invariant subspace of $L^{2}$ for $j=1,2, \ldots$ and $\mathcal{M}_{j} \times \mathcal{M}_{\ell}=\left\{f g ; f \in \mathcal{M}_{j}\right.$ and $\left.g \in \mathcal{M}_{\ell}\right\}=\langle 0\rangle$ if $j \neq \ell$. If $\mathcal{M}=$ $\sum_{j=1}^{\infty} \oplus \mathcal{M}_{j}$, then $\mathcal{M}$ is a weakly divisible invariant subspace.

Proof. If $f \in \mathcal{M}$, then $f=\sum_{j=1}^{\infty} f_{j}$ and $|f(z)|=\sum_{j=1}^{\infty}\left|f_{j}(z)\right|(z \in D)$ by hypothesis. This implies that $\mathcal{M}$ is weakly divisible.

Corollary 4.6. Let $1 \leqslant \ell \leqslant \infty$. Suppose $D_{j}$ is an open set in $D$ with $\mu\left(\partial D_{j}\right)=0$ for $1 \leqslant j \leqslant \ell, D_{i} \cap D_{j}=\emptyset(i \neq j)$ and $D=\bigcup_{j=1}^{\ell} D_{j}$. Then $\mathcal{M}=\sum_{j=1}^{\ell} \oplus L_{a}^{2}\left(D_{j}\right)$ is weakly divisible.

Proof. This is a result of Corollary 4.4 and Proposition 4.5.

Proposition 4.7. If $\mathcal{M}$ is a weakly divisible invariant subspace of $L^{2}$ and $\varphi$ is a unimodular function in $L^{\infty}$, then $\varphi \mathcal{M}$ is a weakly divisible invariant subspace.

Proof. From the definition of weak divisibility, the proposition follows trivially.

Corollary 4.8. If $\varphi$ is a unimodular function in $L^{\infty}$, then $\varphi L_{a}^{2}$ is weakly divisible.

Acknowledgements. This research was partly supported by Grant-in-Aid for Scientific Research, Ministry of Education of Japan.

\section{References}

1. S. Axler AND P. Bourdon, Finite-codimensional invariant subspaces of Bergman spaces, Trans. Am. Math. Soc. 306 (1988), 805-817.

2. A. Beurling, On two problems concerning linear transformations in Hilbert space, Acta Math. 81 (1948), 239-255.

3. N. DAS, The kernel of a Hankel operator on the Bergman space, Bull. Lond. Math. Soc. 31 (1999), 75-80.

4. T. W. Gamelin, Uniform algebras (Prentice Hall, Englewood Cliffs, NJ, 1984).

5. H. Hedenmalm, B. Korenblum and K. Zhu, Theory of Bergman Spaces, Graduate Texts in Mathematics, vol. 199 (Springer, 2000).

6. T. NAKAZI AND T. OsAWA, Finite-rank intermediate Hankel operators on the Bergman space, Int. J. Math. Math. Sci. 25 (2001), 19-31.

7. E. Strouse, Finite rank intermediate Hankel operators, Arch. Math. 67 (1996), 142-149. 